\title{
Correction to: Zooplankton indicator-based assessment in relation to site location and abiotic factors: a case study from the Gulf of Riga
}

\author{
Astra Labuce $(\mathbb{D})$ Inta Dimante-Deimantovica $(\mathbb{D}$ • \\ Juris Tunens (D) Solvita Strake (D)
}

Published online: 17 June 2020

(C) Springer Nature Switzerland AG 2020

Correction to: Environ Monit Assess (2020) 192: 147 https://doi.org/10.1007/s10661-020-8113-9

This paper, published in Environ Monit Assess 192, 147 (2020), contains errors in Table 5. The corrected table is provided below. The errors were small (inaccurately indicated column names for columns 4,7 , and 10 and site titles for rows 6 and 7) and the corrections do not alter in any way the conclusions of the article.

Publisher's note Springer Nature remains neutral with regard to jurisdictional claims in published maps and institutional affiliations.

The online version of the original article can be found at https://doi.org/10.1007/s10661-020-8113-9

A. Labuce $(\bowtie) \cdot$ I. Dimante-Deimantovica $\cdot$ J. Tunens • S. Strake

Latvian Institute of Aquatic Ecology, Agency of Daugavpils

University, Voleru str. 4, Riga LV-1007, Latvia

e-mail: astra.labuce@1hei.lv

I. Dimante-Deimantovica

Norwegian Institute for Nature Research, Gaustadalléen 21,

NO-0349 Oslo, Norway 
Table 5 Mean Size and Total Stock (MSTS)-based assessment for period 2012-2017 of the Gulf of Riga (GoR). For sites location, see Fig. 1

\begin{tabular}{|c|c|c|c|c|c|c|c|c|c|c|c|}
\hline \multirow[t]{3}{*}{ Site } & \multirow{2}{*}{\multicolumn{3}{|c|}{ GES thresholds }} & \multicolumn{8}{|c|}{ Assessment (2012-2017) } \\
\hline & & & & \multicolumn{3}{|l|}{ Mean } & \multicolumn{3}{|c|}{${ }_{\mathrm{L}} \mathrm{CI} 99 \%$} & \multirow{2}{*}{$\begin{array}{l}\text { Decision } \\
\text { Mean }\end{array}$} & \multirow[b]{2}{*}{${ }_{\mathrm{L}} \mathrm{CI} 99 \%$} \\
\hline & MS & TZB & TZA & MS & TZB & TZA & MS & TZB & TZA & & \\
\hline $\mathrm{C} 1$ & 2.29 & 303.3 & 93.2 & 4.10 & $299.3^{\mathrm{b}}$ & $96.2^{\mathrm{b}}$ & 1.72 & 96.1 & 30.1 & GES & Sub-GES \\
\hline $\mathrm{C} 2$ & 2.39 & 282.7 & 86.6 & 3.85 & $303.5^{\mathrm{b}}$ & $103.4^{\mathrm{b}}$ & 0.98 & 114.1 & 47.8 & GES & Sub-GES \\
\hline $\mathrm{C} 3$ & 2.02 & 108.3 & 25.7 & 3.14 & 394.8 & 139.5 & 2.16 & 176.5 & 61.3 & GES & GES \\
\hline $\mathrm{C} 4$ & 1.23 & 102.3 & 18.8 & 4.07 & 313.6 & 98.7 & 1.54 & 117.7 & 41.3 & GES & GES \\
\hline Coastal & 2.54 & 258.3 & 54.7 & 3.79 & $321.8^{\mathrm{b}}$ & 110.9 & 1.62 & 155.8 & 54.0 & GES & Sub-GES \\
\hline $\mathrm{O} 1$ & 2.90 & 154.7 & 36.3 & 5.66 & 253.5 & 61.2 & 3.32 & 140.8 & 33.7 & GES & Sub-GES \\
\hline $\mathrm{O} 2$ & 2.81 & 76.9 & 19.2 & 5.23 & 351.8 & 73.6 & 3.03 & 195.9 & 41.4 & GES & GES \\
\hline Open & 2.89 & 130.1 & 32.1 & 5.45 & 333.2 & 80.4 & 3.62 & 179.0 & 40.5 & GES & GES \\
\hline GoR & 3.09 & 209.2 & 47.5 & 4.62 & 305.8 & 92.5 & 2.80 & 191.3 & 49.0 & GES & Sub-GES \\
\hline
\end{tabular}

Sub-GES cases are italicized

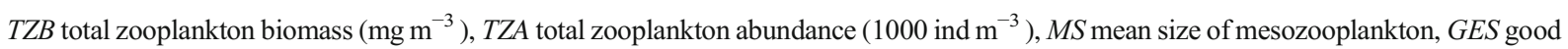
environmental status, ${ }_{L}$ CI 99\% lower $99 \%$ confidence interval.

${ }^{\mathrm{b}}$ CuSum dropped below GES threshold value during 2012-2017 (Fig. 5) 